\title{
SNAP telescope: an update
}

\section{Michael L. Lampton, Michael J. Sholl, Michael H. Krim, R. Besuner, Carl W. Akerlof, et al.}

Michael L. Lampton, Michael J. Sholl, Michael H. Krim, R. Besuner, Carl W. Akerlof, Greg Aldering, R. Amanullah, Pierre Astier, Charles Baltay, E. Barrelet, Stephane Basa, Christopher Bebek, John Bercovitz, Lars Bergstrom, Gary Berstein, Manfred Bester, Ralph C. Bohlin, Alain Bonissent, Charles R. Bower, Myron Campbell, William C. Carithers, Eugene D. Commins, C. Day, Susana E. Deustua, Richard S. DiGennaro, Anne Ealet, Richard S. Ellis, William Emmett, Mikael Eriksson, D. Fouchez, Andrew Fruchter, Jean-Francois Genat, Gerson Goldhaber, Ariel Goobar, Donald E. Groom, Henry D. Heetderks, Stephen E. Holland, Dragan Huterer, William E. Johnston, Richard W. Kadel, Armin Karcher, Alex G. Kim, William F. Kolbe, Robin E. Lafever, J. I. Lamoureux, Oliver LeFevre, Michael E. Levi, Daniel S. Levin, Eric V. Linder, Stewart C. Loken, Roger Malina, Alain Mazure, Timothy A. McKay, Shawn P. McKee, Ramon Miquel, Nicholas Morgan, E. Mortsell, Nick Mostek, Stuart Mufson, J. A. Musser, Peter E. Nugent, Hakeem M. Oluseyi, Reynald Pain, Nick P. Palaio, David H. Pankow, Saul Perlmutter, Eric Prieto, David Rabinowitz, Alexandre Refregier, Jason Rhodes, Natalie A. Roe, Michael S. Schubnell, G. Smadja, R. Smith, George F. Smoot, Jeffrey A. Snyder, Anthony Spadafora, Andrew Szymkowiak, Gregory Tarle, Keith Taylor, A. Tilquin, Andrew D. Tomasch, D. Vincent, Henrik von der Lippe, Jean-Pierre Walder, Guobin Wang, "SNAP telescope: an update," Proc. SPIE 5166, UVI Optical/IR Space Telescopes: Innovative Technologies and Concepts, (30 January 2004); doi: $10.1117 / 12.502333$ 


\title{
SNAP Telescope: an Update
}

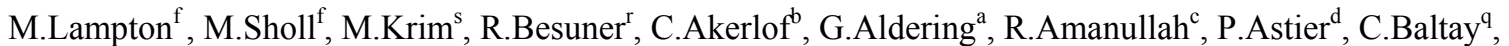
E.Barrelet $^{\mathrm{d}}$, S.Basa ${ }^{\mathrm{m}}$, C.Bebek ${ }^{\mathrm{a}}$, J.Bercovitz ${ }^{\mathrm{a}}$, L.Bergstrom ${ }^{\mathrm{c}}$, G.Berstein ${ }^{\mathrm{e}}$, M.Bester ${ }^{\mathrm{f}}$, R.Bohlin ${ }^{\mathrm{k}}$, A.Bonissent ${ }^{\mathrm{m}}$, C.Bower ${ }^{\mathrm{h}}$, M.Campbell ${ }^{\mathrm{b}}$, W.Carithers ${ }^{\mathrm{a}}$, E.Commins ${ }^{\mathrm{f}}$, C.Day ${ }^{\mathrm{a}}$, S.Deustua ${ }^{\mathrm{i}}$, R.DiGennaro ${ }^{\mathrm{a}}$, A.Ealet ${ }^{\mathrm{m}}$, R.Ellis', W.Emmett ${ }^{\mathrm{q}}$, M.Eriksson ${ }^{\mathrm{c}}$, D.Fouchez ${ }^{\mathrm{m}}$, A.Fruchter ${ }^{\mathrm{k}}$, J-F.Genat ${ }^{\mathrm{d}}$, G.Goldhaber ${ }^{\mathrm{a}}$, A.Goobar ${ }^{\mathrm{c}}$, D.Groom ${ }^{\mathrm{a}}$, H.Heetderks ${ }^{\mathrm{f}}$, S.Holland ${ }^{\mathrm{a}}$, D.Huterer ${ }^{\mathrm{a}}$, W.Johnston ${ }^{\mathrm{a}}$, R.Kadel ${ }^{\mathrm{a}}$, A.Karcher ${ }^{\mathrm{a}}$, A.Kim ${ }^{\mathrm{a}}$, W.Kolbe ${ }^{\mathrm{a}}$, R.Lafever ${ }^{\mathrm{a}}$, J.Lamoureaux ${ }^{\mathrm{a}}$, O.Lefevre ${ }^{\mathrm{a}}$, M.Levi ${ }^{\mathrm{a}}$, D.Levin ${ }^{\mathrm{b}}$, E.Linder $^{\mathrm{a}}$, S.Loken ${ }^{\mathrm{a}}$, R.Malina ${ }^{\mathrm{m}}$, A.Mazure $^{\mathrm{m}}$, T.McKay ${ }^{\mathrm{b}}$, S.McKee ${ }^{\mathrm{b}}$, R.Miquel ${ }^{\mathrm{a}}$, N.Morgan ${ }^{\mathrm{q}}$, E.Mortsell ${ }^{\mathrm{c}}$, N.Mostek ${ }^{\mathrm{h}}$, S.Mufson ${ }^{\mathrm{h}}$, J.Musser $^{\mathrm{h}}$, P.Nugent ${ }^{\mathrm{a}}$, H.Oluseyi ${ }^{\mathrm{a}}$, R.Pain ${ }^{\mathrm{d}}$, N.Palaio ${ }^{\mathrm{a}}$, D.Pankow ${ }^{\mathrm{f}}$, S.Perlmutter ${ }^{\mathrm{a}}$, E.Prieto ${ }^{\mathrm{m}}$, D.Rabinowitz ${ }^{\mathrm{q}}$,

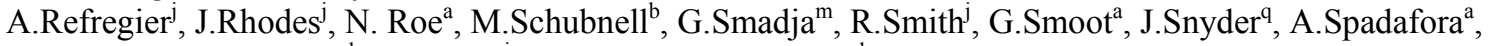
A.Szymkowiak ${ }^{\mathrm{q}}$, G.Tarle ${ }^{\mathrm{b}}$, K.Taylor ${ }^{\mathrm{j}}$, A.Tilquin ${ }^{\mathrm{m}}$, A.Tomasch ${ }^{\mathrm{b}}$, D.Vincent ${ }^{\mathrm{g}}$, H.von der Lippe ${ }^{\mathrm{a}}$, J-P.Walder ${ }^{\mathrm{a}}$, G.Wang ${ }^{\mathrm{a}}$

\author{
${ }^{a}$ Lawrence Berkeley National Laboratory \\ ${ }^{\mathrm{b}}$ University of Michigan, Ann Arbor MI, USA \\ ${ }^{\mathrm{c}}$ University of Stockholm, Stockholm, Sweden \\ ${ }^{\mathrm{d}}$ CNRS/IN2P3/LPNHE, Paris, France \\ ${ }^{\mathrm{e}}$ University of Pennsylvania, Philadelphia, PA USA \\ ${ }^{\mathrm{f}}$ University of California, Berkeley, CA USA \\ ${ }^{g}$ CNRS/IN2P3/CPPM, Marseille, France \\ ${ }^{\mathrm{h}}$ Indiana University, Bloomington, IN, USA \\ ${ }^{\mathrm{i}}$ American Astronomical Society, Washington, DC, USA \\ ${ }^{\mathrm{j}}$ California Institute of Technology, Pasadena, CA, USA \\ ${ }^{\mathrm{k}}$ Space Telescope Science Institute, Baltimore, MD, USA \\ ${ }^{1}$ Case Western Reserve University, Cleveland, OH,USA \\ ${ }^{\mathrm{m}} \mathrm{CNRS} / \mathrm{INSU} / \mathrm{LAM}$, Marseille, France \\ ${ }^{\mathrm{n}}$ Cambridge University, Cambridge UK \\ ${ }^{\circ}$ NASA Goddard Space Flight Center, Greenbelt MD, USA \\ ${ }^{\mathrm{p}} \mathrm{CNRS} / \mathrm{IN} 2 \mathrm{PL}$, Lyon, France \\ ${ }^{q}$ Yale University, New Haven, CT, USA \\ ${ }^{\mathrm{r}}$ Robert Besuner Consulting, Madera CA, USA \\ ${ }^{\mathrm{s}}$ Michael Krim Consulting, Trumbull CT, USA
}

\begin{abstract}
We present the baseline telescope design for the telescope for the SuperNova/Acceleration Probe (SNAP) space mission. SNAP's purpose is to determine expansion history of the Universe by measuring the redshifts, magnitudes, and spectral classifications of thousands of supernovae with unprecedented accuracy. Discovering and measuring these supernovae demand both a wide optical field and a high sensitivity throughout the visible and near IR wavebands. We have adopted the annular-field three-mirror anastigmat (TMA) telescope configuration, whose classical aberrations (including chromatic) are zero. We show a preliminary optmechanical design that includes important features for stray light control and on-orbit adjustment and alignment of the optics. We briefly discuss stray light and tolerance issues, and present a preliminary wavefront error budget for the SNAP Telescope. We conclude by describing some of the design tasks being carried out during the current SNAP research and development phase.
\end{abstract}

Keywords: three-mirror telescopes, space astronomy, wide-field imaging 


\section{SNAP MISSION OVERVIEW}

The SuperNova/Acceleration Probe (SNAP) is a planned satellite experiment designed to precisely measure the expansion history of the universe. The experiment is motivated by the remarkable discovery of an accelerating expansion rate ${ }^{1,2}$. This acceleration suggests that the universe contains some form of dark energy, which can be modeled by its equation of state at the present epoch $\mathrm{w}_{0}$ and its rate of change with redshift w'. To effectively test models of the expansion, it is essential to compare accurate observational data against model predictions of the expansion rate as a function of lookback time, or equivalently as a function of redshift. Type Ia supernovae populate the observable universe and serve as accurately standardizable candles. Each measured supernova furnishes a redshift and a peak magnitude. The redshift is a measure of the expansion of the universe between its epoch and the present, while the peak magnitude is a measure of the elapsed time since the supernova event. Properly calibrated and sorted into systematic classes, a collection of a few thousand such supernovae spanning the redshift range $0.1<\mathrm{z}<1.7$ will provide important new constraints on models of the universe and the dark energy that it contains. Independent constraints on the evolution of the universe can be obtained from the observation of weak gravitational lensing of background galaxies by foreground mass concentrations; accordingly weak lensing studies are also part of the SNAP investigation. Descriptions of the mission and its science are presented at the SNAP home page, http://snap.lbl.gov, and elsewhere in the literature. ${ }^{3,4,5}$ The SNAP telescope design and status was summarized in an earlier report by Lampton et al. ${ }^{6}$ Here, we briefly review that baseline design and describe the ongoing work planning the manufacture and test of the telescope.

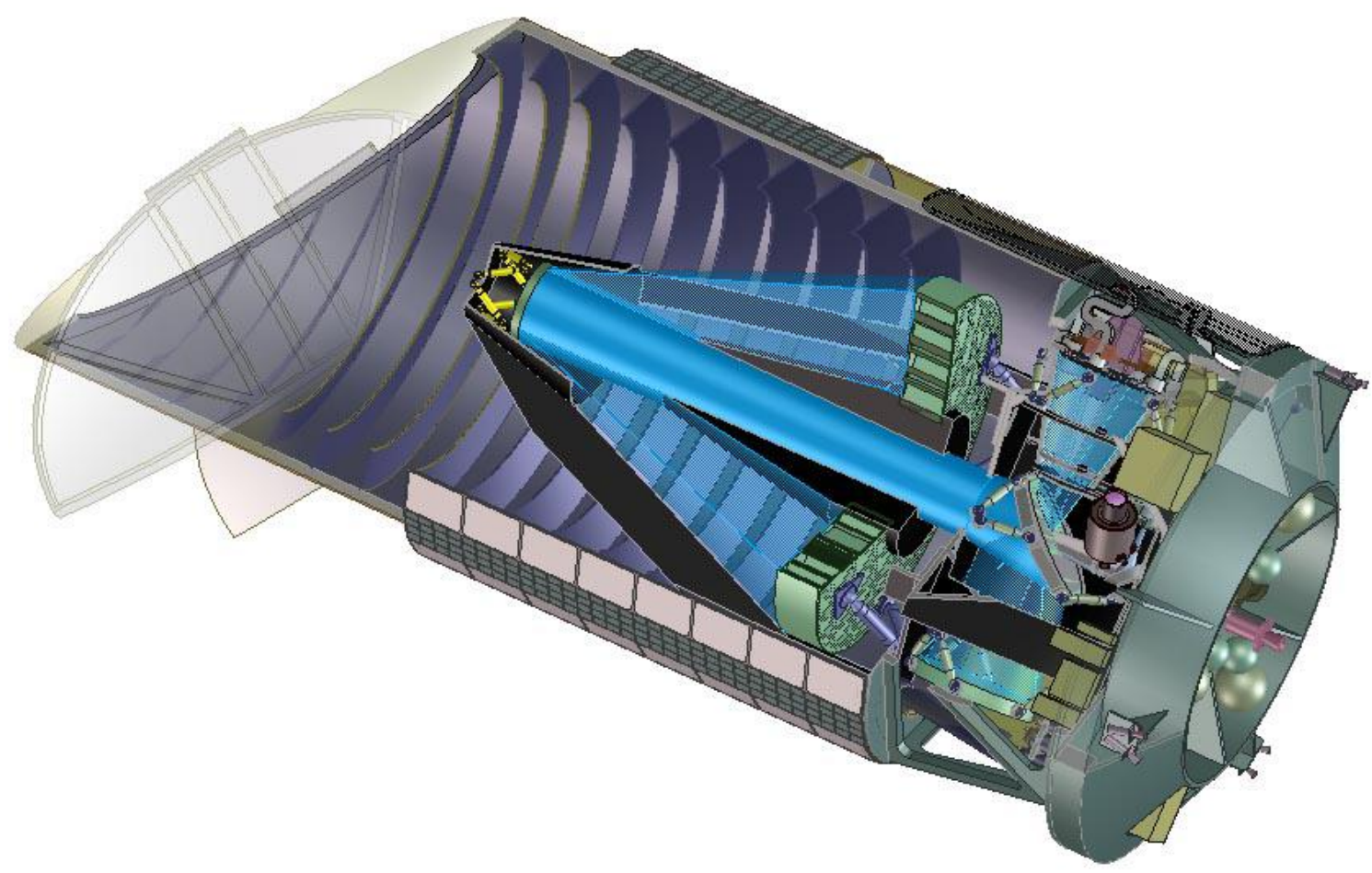

Fig. 1: Cutaway view of SNAP. The entire telescope attaches to the spacecraft structure at right by means of structural bipods. The outer baffle also attaches to the spacecraft structure by means of its separate supporting struts. A hinged split door, shown open in light gray, protects the cleanliness of the optics until on-orbit commissioning begins. Solar panels are fixed to the sunward side of the spacecraft (underside in this illustration) and are not deployed. A large thermal radiator on the antisunward side provides passive cooling for the detector cryostat (upper right). The cryostat carries 72 image sensors for multiband survey work and a low-dispersion spectrometer for targeted follow-up. 


\section{MISSION REQUIREMENTS}

Since SNAP's inception in 1999 a variety of mission scenarios have been explored to discover what combinations of sky survey area, telescope aperture, wavelength coverage, and observing plan yield the best distribution of supernovae in the sense of optimizing constraints on parameters that describe the expansion history of the universe. From these simulations a consistent "reference model" has emerged. Its characteristics are summarized in Table 1.

Table 1: SNAP Reference Model

\begin{tabular}{|c|c|}
\hline Wavelength range & 0.35 to 1.7 microns \\
\hline Telescope aperture & 2.0 meters \\
\hline Working field of view & 0.7 square degrees \\
\hline Survey field size & 15 square degrees \\
\hline Field revisit period & 4 days \\
\hline Fraction of observing time, survey & $60 \%$ \\
\hline Fraction of observing time, spectroscopy & $40 \%$ \\
\hline
\end{tabular}

In support of this model we have studied a variety of mission operational scenarios. A mission description has evolved that delivers the resources needed to accommodate the reference model, including considerations of passive thermal design, stray light from the Sun and the Earth, survey field visibility throughout the orbit and throughout the year, orbit stability and accessibility, detector pixel sizes, focal length, sky coverage, and many other practical factors and constraints summarized in Table 2.

Table 2: SNAP Mission Characteristics

\begin{tabular}{|c|c|}
\hline Orbit perigee, apogee & $2.6 \times 24.9$ Re geocentric \\
\hline Orbit period & 3.00 days \\
\hline Orbit inclination & coplanar with moon \\
\hline Telescope aperture and speed & 2 meters; f/1.25 primary, f/10.83 final \\
\hline Effective focal length & 21.66 meters \\
\hline Field of view size & $1.5 \mathrm{deg}$ diameter annulus \\
\hline Angle between view and sun & 70 to 110 degrees \\
\hline Survey regions & near $\mathrm{N}$ and/or $\mathrm{S}$ ecliptic poles \\
\hline Overall observatory length & 6 meters \\
\hline Optical telescope length & $\sim 3$ meters \\
\hline Overall observatory mass & $1600 \mathrm{~kg}$ \\
\hline Detectors, visible & silicon CCDs, 10.5 um pixels \\
\hline Detectors, NIR & HgCdTe arrays, 18 um pixels \\
\hline
\end{tabular}

\section{MISSION EXECUTION PLAN}

We have identified two primary science survey fields. These are located near the north and south ecliptic poles where natural Zodiacal light is minimized for best near IR sensitivity and for visibility throughout the year owing to the nearly constant angle between the target field and the sun. In a high Earth orbit, a low orbital inclination serves to keep the Earth and moon also nearly at right angles to our view direction. We utilize this viewing geometry in several ways. First, the solar panels can be rigidly body-mounted on the sunward side of the spacecraft, which avoids the cost, failure modes, and structural flexibility of deployed panels. Second, the passive cooling radiator can be rigidly located on the antisunward side of the spacecraft, in permanent shadow. Third, the stray light baffling can be optimized for a limited range of solar roll and elevation angles, and for a limited range of Earth elevation angles. We plan to have the 
spacecraft perform 90 degree roll maneuvers every 3 months during the mission to keep up with the mean ecliptic longitude of the sun. The detector array has a 90 degree roll symmetry that allows its photometric data acquisition to continue from season to season.

The mission will be conducted by repeatedly scanning a 7.5 square degree zone near the north ecliptic pole for a 16 month period, and later conducting a similar study near the south ecliptic pole. During each study, scans will repeat with a four day cadence. Each scan will provide photometry in nine bands spanning the visible and near IR for all objects in the zone down to a faint magnitude limit of about $A B=27.5$. In this way, the light curve for each detected supernova will be determined in order to provide magnitude and classification data. During each four day period, time will also be taken to perform follow-up spectroscopy on each detected supernova near its maximum light to determine its redshift and obtain further classification data. To carry out these measurements, two instruments share a common focal plane. First, a large passively cooled multiband imager with approximately 600 million pixels occupies most of the focal plane area; this is described by Lampton et $\mathrm{al}^{7}$ and Bebek et $\mathrm{al}^{8}$. Follow-up spectroscopy on selected targets is conducted using a high-efficiency low-dispersion spectrometer equipped with an integral field unit described by Ealet et al ${ }^{9}$.

\section{OPTICAL CONFIGURATION}

The SNAP mission requires a telescope that provides a wide field of view with diffraction limited imaging performance, yet it must deliver a long focal length and a flat achromatic image surface within a short overall length to fit within the allowed payload envelope. To achieve a focal length of 22 meters within a package length of 3 meters demands a telephoto ratio of about 1/7. These combined requirements are not met by conventional telescope designs such as the Cassegrain, Ritchey-Chretien, Paul-Baker, etc (for a detailed discussion see Lampton et $\mathrm{al}^{6}$ ), but are easily met by the three-mirror anastigmat (TMA) configuration developed by Korsch ${ }^{10}$. In particular we adopt Korsch's on-axis annular-field configuration which uses a flat folding mirror that rotates the final focal surface away from the primary mirror axis, placing it to one side, thereby allowing free access to a large focal plane instrument. In this side location the detector is moved away from the primary optical axis so that the detector no longer blocks any secondary light. For SNAP such an advantage is mandatory since our detector is larger than our secondary mirror. This configuration also provides a natural way to obtain passive detector cooling, since one side of the telescope will always face the antisunward direction. A schematic view is shown in Figure 2.

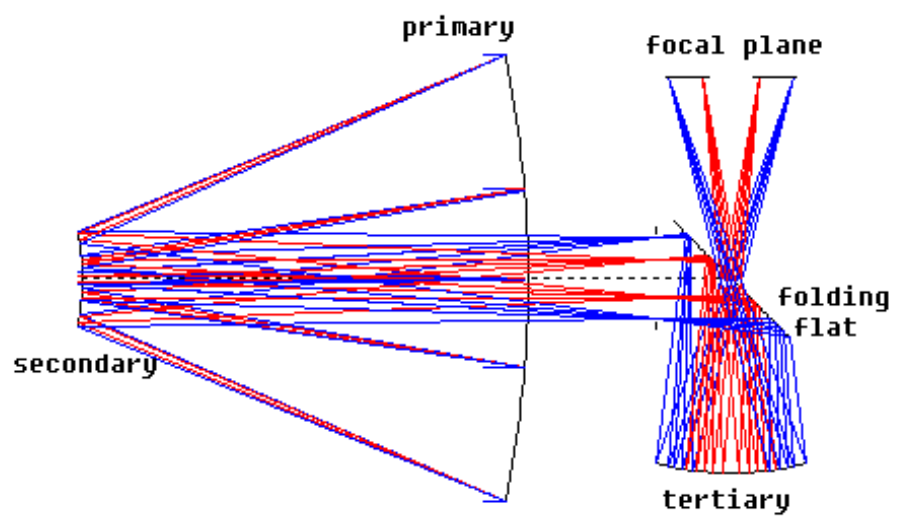

Fig. 2: Schematic SNAP optics layout. The entrance pupil is defined by the primary mirror. A field stop is located behind the primary mirror (small vertical marks) for stray light control. The exit pupil is at the folding mirror.

A specific baseline optical configuration has been developed by ray tracing a generalized TMA with the design parameters chosen by a nonlinear least squares method. In this way a specific target focal length and working field size can be specified, and alternative designs can be compared. The design parameters 
that control the aberrations are the three powered mirror curvatures, the three conic constants, and the three spacings (primary to secondary, secondary to tertiary, and tertiary to focal plane). These nine parameters are adjustable, subject to several constraints. The effective focal length (EFL), the primary aperture, and the focal plane diameter we regard as given. The element spacings and the consequent locations of the field stop and the exit pupil have to be carefully managed to achieve a satisfactory package and to place the exit pupil within the central hole of the folding mirror. The mirrors are pure conic sections of revolution having no polynomial or cylinder terms. Full details, along with spot diagrams and other diagnostics, are listed in Lampton et $\mathrm{al}^{6}$. The optical system and its performance are summarized in Tables 3 and 4.

Table 3: Optical Prescription

\begin{tabular}{|l|l|l|l|l|c|c|}
\hline & $\begin{array}{l}\text { Diameter, } \\
\text { meters }\end{array}$ & $\begin{array}{l}\text { Central hole, } \\
\text { meters }\end{array}$ & $\begin{array}{l}\text { Curvature, } \\
\text { recip meters }\end{array}$ & Asphericity & $\begin{array}{l}\text { Xlocation, } \\
\text { meters }\end{array}$ & $\begin{array}{l}\text { Zlocation, } \\
\text { meters }\end{array}$ \\
\hline Primary & 2.00 & 0.5 & -0.2037466 & -0.981128 & 0 & 0 \\
\hline Secondary & 0.45 & none & -0.9099607 & -1.847493 & 0 & -2.00 \\
\hline Folding flat & $0.66 \times 0.45$ & $0.19 \times 0.12$ & 0 & 0 & 0 & +0.91 \\
\hline Tertiary & 0.68 & none & -0.7112388 & -0.599000 & -0.87 & +0.91 \\
\hline Focal plane & 0.567 & 0.258 & 0 & 0 & +0.9 & +0.91 \\
\hline
\end{tabular}

Table 4: Telescope Parameters Summary

\begin{tabular}{|c|c|}
\hline Focal Length & 21.66 meters \\
\hline Aperture & 2.0 meters \\
\hline Final focal ratio & $\mathrm{f} / 10.83$ \\
\hline Optical field & Annular, 6 to $13 \mathrm{mrad} ; 1.37 \mathrm{sq} \mathrm{deg}$ \\
\hline RMS geometric blur & 2.8 microns, average 1 dimension \\
\hline Central obstruction & $16 \%$ area when fully baffled \\
\hline Vane obstruction & $4 \%$ area, tripod or quadrupod \\
\hline
\end{tabular}

The departure of any surface from its nominal mathematical conic section, or the misplacement or misorientation of any of the surfaces, causes a wavefront error and a degraded image quality. One measure of this degradation is the telescope's Strehl ratio, which is the peak monochromatic image irradiance divided by the theoretical peak irradiance for the ideal diffraction limited image. Strehl ratio can be converted into RMS wavefront error (RMS WFE) through Marechal's relation. To achieve a system Strehl ratio of 0.77 at 0.633 microns wavelength, the total WFE must be kept to about $50 \mathrm{~nm}$ rms. This allowed WFE can be apportioned into individual contributions for planning purposes. A very preliminary apportionment is listed in Table 5.

Table 5: Wavefront Error Budget

\begin{tabular}{|l|c|c|c|c|c|}
\hline Optical Element & $\begin{array}{c}\text { Manufacturing } \\
(\text { Figure) Error } \\
(\mathrm{nm})\end{array}$ & $\begin{array}{c}\text { Mount } \\
\text { Distortions } \\
(\mathrm{nm})\end{array}$ & $\begin{array}{c}\text { Misalignment } \\
(\mathrm{nm})\end{array}$ & $\begin{array}{c}\text { Long-term } \\
\text { Stability } \\
(\mathrm{nm})\end{array}$ & $\begin{array}{c}\text { Gravity } \\
\text { Release (nm) }\end{array}$ \\
\hline Primary & 20 & 8 & -- & 10 & 20 \\
\hline Secondary & 15 & 8 & 15 & 10 & 10 \\
\hline Fold Mirror & 12 & 6 & -- & -- & -- \\
\hline Tertiary & 15 & 8 & 6 & 6 & 6 \\
\hline Structure & -- & -- & -- & -- & 8 \\
\hline Root Sum Square (RSS) & 31.5 & 15.1 & 17.2 & 16.5 & 24.5 \\
\hline
\end{tabular}

\begin{tabular}{|l|c|}
\hline Margin $(\mathrm{nm})$ & 15 \\
\hline Total RSS $(\mathrm{nm})$ & 51.1 \\
\hline Strehl, at $0.633 \mu \mathrm{m}$ & 0.77 \\
\hline
\end{tabular}


Table 5 details an apportionment of Optical Path Difference (OPD) errors for various elements of the SNAP telescope. OPD errors are measured in the pupil plane as Root Mean Square (RMS) deviations from ideal produced by various error contributors. Manufacturing (figure) error is the deviation between the asmanufactured primary mirror surface figure, and the optical prescription, multiplied by two to account for the doubling effect of the mirrors. Mount distortions are produced by clamping loads, material dissimilarities (thermal and mechanical), misalignment-induced torque, and other physical effects of the glass-structure mounting hardware. Misalignment refers to OPD errors produced by non-ideal positioning of a given optical element; they do not have a one to one relationship with mechanical tolerances. Only OPD errors which cannot be corrected via motion of the secondary hexapod are considered. Long-term stability refers primarily to the effects of thermal distortion, but includes any long-timescale structural changes. Gravity release terms are those effects not removed or by gravity unloaders during optical testing.

The image quality of the telescope is driven in part by the SNR requirement, and also by the potential systematic supernova spectrum contamination by unwanted light from the supernova host galaxy. We have presently baselined a system Strehl ratio of 0.90 at one micron wavelength, corresponding to an RMS wavefront error (WFE) of $51 \mathrm{~nm}$, or a Strehl ratio of 0.77 at the commonly used test wavelength of 0.633 microns. Diffraction, aberrations, and mirror surface figure are but three contributors to the overall system image quality, discussed more fully below.

\section{SYSTEM LEVEL PERFORMANCE}

The optical performance is but one of the factors that control the recovered image quality and the resulting photometric signal to noise ratio. The SNAP spacecraft and payload elements other than the telescope contribute to the image blur as well. In Figure 3 below we have summarized the principal blur contributors and compare these with our detector array pixel sizes, shown as heavy bars.

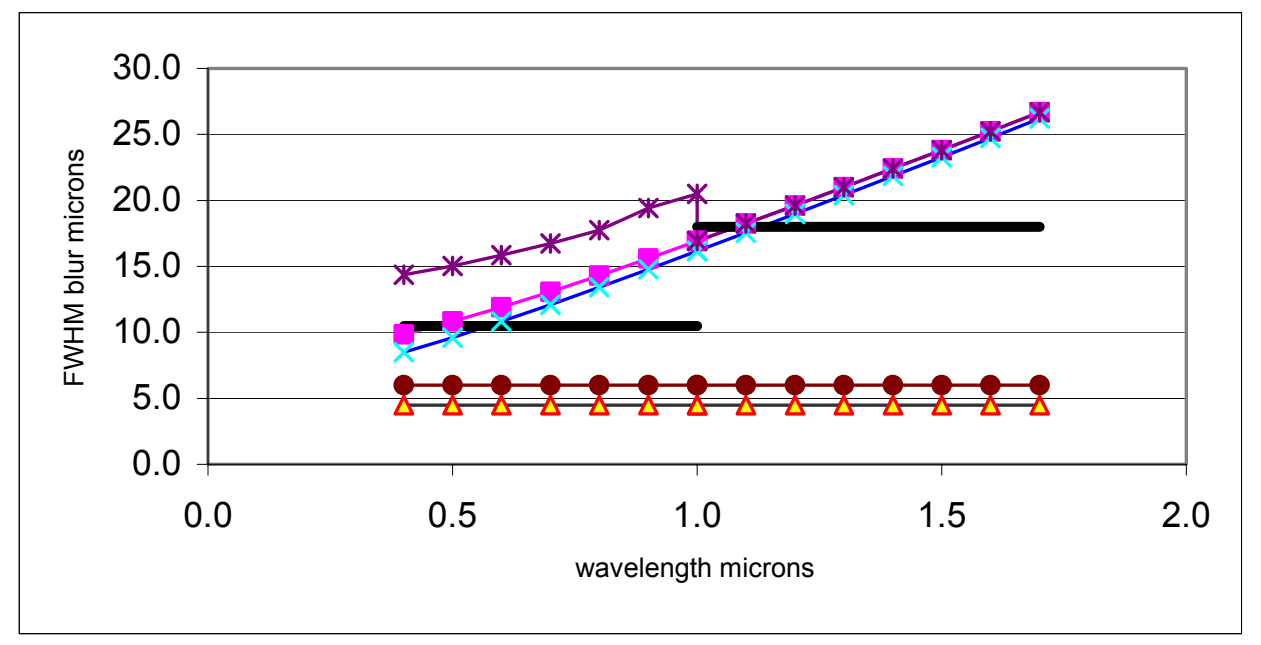

Figure 3: FWHM point-spread function estimates. Curves, bottom to top: 1: attitude control system (ACS) jitter; 2: ideal optical aberrations averaged over the annular field; 3 : total optical = aberrations + diffraction; 4: optical + ACS; 5: curve $4+$ silicon diffusion standard deviation $\sigma=4 \mu \mathrm{m}$. Heavy bars: pixel sizes for silicon and $\mathrm{HgCdTe}$ image sensors.

Figure 3 shows that, at NIR wavelengths, the major contributor to our point spread function is diffraction by the circular aperture obstructed by the central secondary mirror and baffle and by the secondary support metering structure. Expected diffraction plots were presented by Lampton et $\mathrm{al}^{6}$. At visible wavelengths the situation is more complicated. Electron-hole diffusion in the fully-depleted high resistivity silicon CCD 
detectors is presently the major term, and optimizing these sensors is a major element of the continuing $R \& D$ effort (Bebek et al, this conference).

In order that the signal-to-noise ratio for photometry not be degraded by the pixel response function, the pixel size should be comparable to or smaller than the total FWHM of the stellar images, plotted above. The use of smaller pixel sizes in the silicon band and larger pixels for the NIR clearly helps us satisfy this consideration. Precision photometry also requires sampling the image at least twice per FWHM. This will be achieved by $2 \times 2$ dithering. Bernstein ${ }^{11}$ demonstrates that a pixel scale near the FWHM, with dithering, optimizes the speed of a photometric survey when detector or telemetry costs place limits on the number of pixels in the focal plane.

For precision image reconstruction, it is important to sample the image at a sufficiently high spatial frequency that essentially all the power in the image is resolved. The traditional sampling rule, based on Nyquist's theorem for band-limited data, is that two samples per cycle at the cutoff frequency are required to reconstruct the data without aliasing. With SNAP, the various blur contributors discussed above act to attenuate the high frequency spatial image power, in addition to the standard pupil-diffraction modulation transfer curve whose cutoff period is $\lambda *$ fnumber. Undersampling, dithering, cosmic ray hits, and many other effects are included in the exposure time calculator developed by Bernstein ${ }^{11}$. The pixel sizes that optimize our SNR are clearly somewhat larger than would be chosen for ideal image reconstruction, but the $2 \times 2$ dithering recovers the Nyquist sampling rate over most of the wavelength range

\section{STRAY LIGHT}

Because SNAP is in nearly perpetual sunlight, and is often illuminated by the full Earth as well, the control of stray light has been a continuing effort. Our goal is to keep all stray light sources far below the natural Zodiacal irradiance level as seen at the focal plane. Our primary concerns are:

- $\quad$ sunlight diffracted around the forward outer baffle edge

- earthshine illuminating the interior of the outer baffle

- off-axis starlight scattering at primary mirror

- light leaks into baffle, aft metering structure, thermal radiator, etc

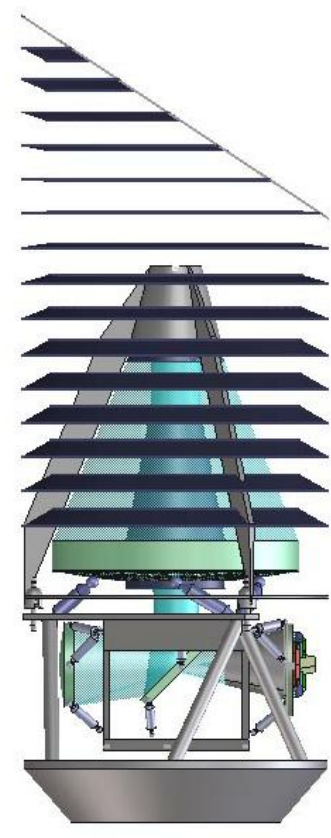

To deal with this first issue will require two successive diffracting edges, with the second lying in the shadow of the first, to yield sufficient attenuation. To handle the second issue we anticipate that the use of inclined baffle blades whose upper surfaces (those that view deep space) are illuminated by the full Earth but whose lower surfaces (those visible to the primary mirror) cannot be directly illuminated by any exterior source. A comprehensive stray light budget is being constructed and will be tracked to guide decisions about the choice of absorbing coatings for the baffle surfaces.

Fig. 4: Schematic treatment of the outer baffle interior vane arrangement. Sunlight is incident from the left, where the height of the baffle and its angled forward edge maintains the baffle interior in darkness. Earthshine is at times incident from the right, however, and therefore the vane angles require particular attention so that the lower vane surfaces (those visible to the primary mirror) are not illuminated by the Earth. 


\section{MECHANICAL CONFIGURATION}

For a space mission it is vital to create a mechanical configuration that provides an extremely stable metering structure that maintains the optical element alignment during ground testing, launch, and orbit operations. The concept adopted for SNAP is a modular one comprising three individually manufactured and verified structural subassemblies that will be brought together during spacecraft/payload integration: a stiff low-precision outer baffle cylinder carrying the exterior solar panels and extensive thermal insulation; a stiff low-precision spacecraft bus structure that carries antennas, batteries, and other major spacecraft support components; and a very stiff deterministic and highly stable telescope structure comprising carbon-fiber metering elements, the kinematically-mounted mirrors, the instrumentation suite, and its own thermal control system. Fig. 5 shows details of the secondary and tertiary metering structures.

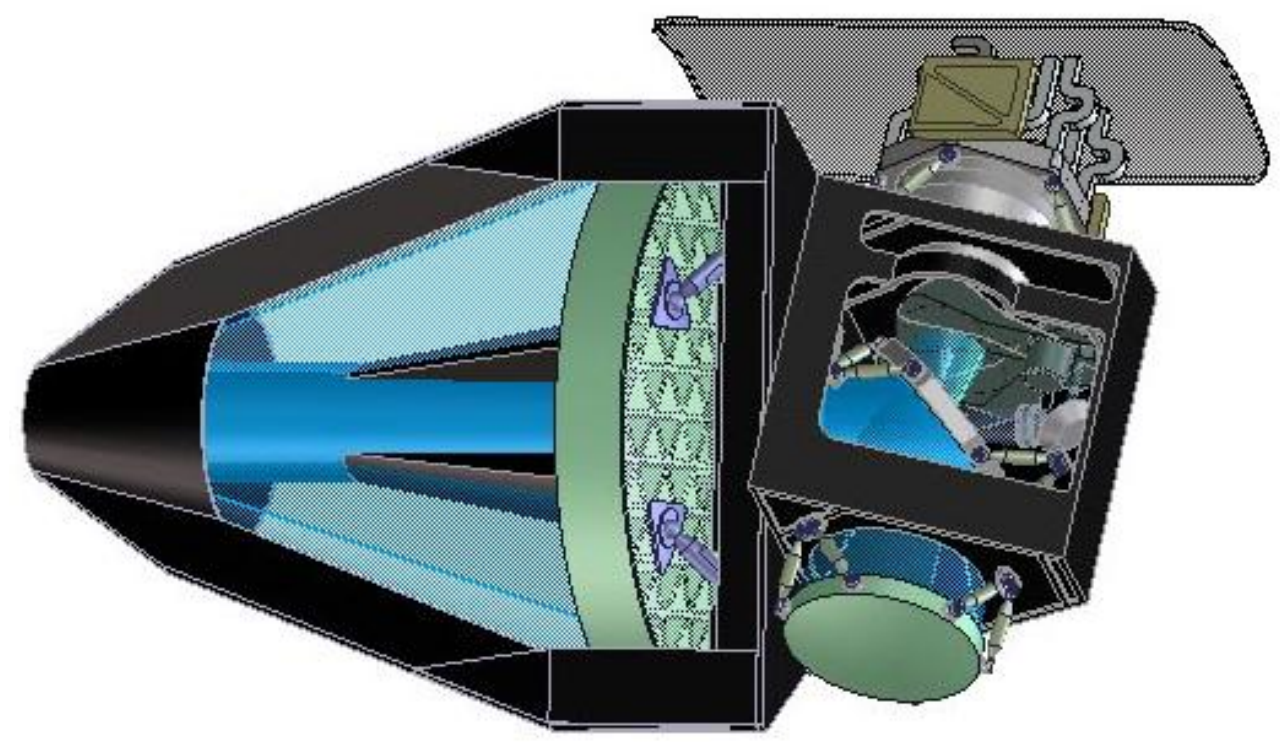

Fig. 5: Telescope metering structure (carbon fiber, shown in dark gray) provides precision control of optical element spacings and orientations. Forward of the primary mirror, the secondary is supported on adjusters within the secondary baffle. Aft of the primary mirror, the tertiary metering structure supports the folding flat, the tertiary, and the focal plane instrumentation. The passive radiator at top is thermally but not structurally linked to the focal plane instrumentation.

Space-proven optical mirror technology is largely based on two approaches: open-back Schott Zerodur glass-ceramic material and Corning ultra-low expansion ULE glass closed-back honeycomb structure. For SNAP either technology has sufficiently low coefficient of thermal expansion and sufficiently well proven lightweight manufacturing techniques. Studies are underway exploring the detailed fabrication and test flows using either process. Alternative materials, including various formulations of silicon carbide, are under study for other space missions and may prove to be competitive for SNAP. We plan to build in a thermal control system that maintains the mirrors within a few degrees $\mathrm{C}$ of the temperature at which their acceptance testing was performed.

We anticipate the need to perform on-orbit focusing and alignment based on ground analysis of the downlinked image quality. Owing to the fast (f/1.25) primary mirror, the location of the secondary mirror is critical, particularly in piston. We plan to include a five-axis motorized a hexapod or other multi-axis positioner into the secondary support structure. The tertiary mirror and folding flat are far less critical but it may nonetheless be advantageous to motorize these mirror positioners also, at least for use in ground alignment. 


\section{INTEGRATION AND TEST PLAN}

We envision the assembly, integration, and testing of the SNAP telescope proceeding through a sequence of stages: the separate telescope optical and mechanical components are individually tested, and then integrated into an OTA which is subjected to further tests to verify its behavior under 1-G conditions and to predict its performance in a zero gravity environment. The buildup of the entire payload continues with the addition of the science cryostat containing the imager and the spectrometer, the outer light baffle, and the spacecraft bus. Qualification testing involves an extended thermal vacuum test, vibration and/or acoustic testing, radio-frequency compatibility and interference tests, prior to shipment to the Cape to mate with the launcher and launch shroud.

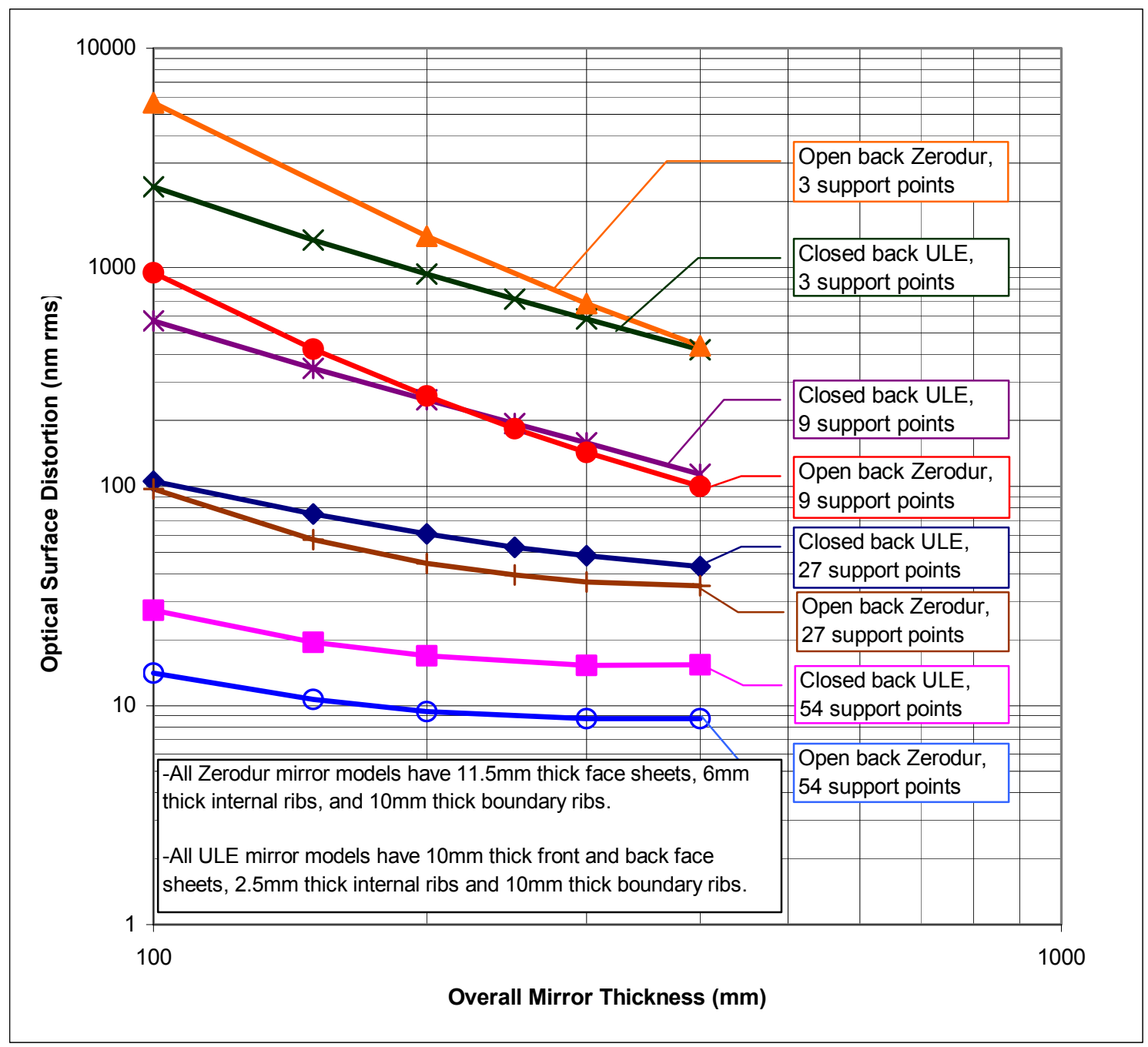

Figure 6: Trend curves for candidate primary mirror FEM distortions in 1-G supported for vertical beam testing by three support bipods (from Besuner et al, in preparation) and with additional support points to distribute the gravity loads for figure acceptance testing. These examples are intended to contrast two alternative commercially feasible designs, namely open-back Zerodur (with realistic face and rib thicknesses) versus closed-back ULE (with more agressive lightweighting). For a thin mirror with only three supports, the inherently stiffer closed-back configuration is advantageous. However, deep mirrors on multipoint supports receive no benefit from the rear face sheet, and the inherently heavier construction of Zerodur open-back mirrors can yield excellent performance. 
Because the telescope will be tested in a one-gravity environment, it is vital to understand the figure errors of the individual mirrors under various orientations under gravity. As a start, we have compiled a variety of mirror and mirror-support deformation predictions, derived from finite-element modeling of the individual mirrors, their mounting bipods, and the precision metering structure.

The ongoing FEM studies are being used to compare prospective mirror materials, lightweighting structure features, and support alternatives. In Figure 6 we show RMS deformations for candidate two-meter aperture primary mirrors of various thicknesses manufactured from Corning ULE glass and from Schott Zerodur. The ULE group has $10 \mathrm{~mm}$ front and rear face sheets, $2.5 \mathrm{~mm}$ thick internal ribs and $10 \mathrm{~mm}$ thick boundary ribs. The Zerodur group is open back with $11.5 \mathrm{~mm}$ thick face sheets, $6 \mathrm{~mm}$ thick internal ribs and $10 \mathrm{~mm}$ thick boundary ribs. For comparison all have triangular isogrid cells with $294 \mathrm{~mm}$ sides. Although the on-orbit primary support will fully kinematic to avoid deforming the mirror by creep of its strongback, during 1-gravity testing it is likely that a large number of supports will be required to demonstrate its figure. Other ongoing studies are exploring various cell sizes, rib thicknesses, and face sheets.

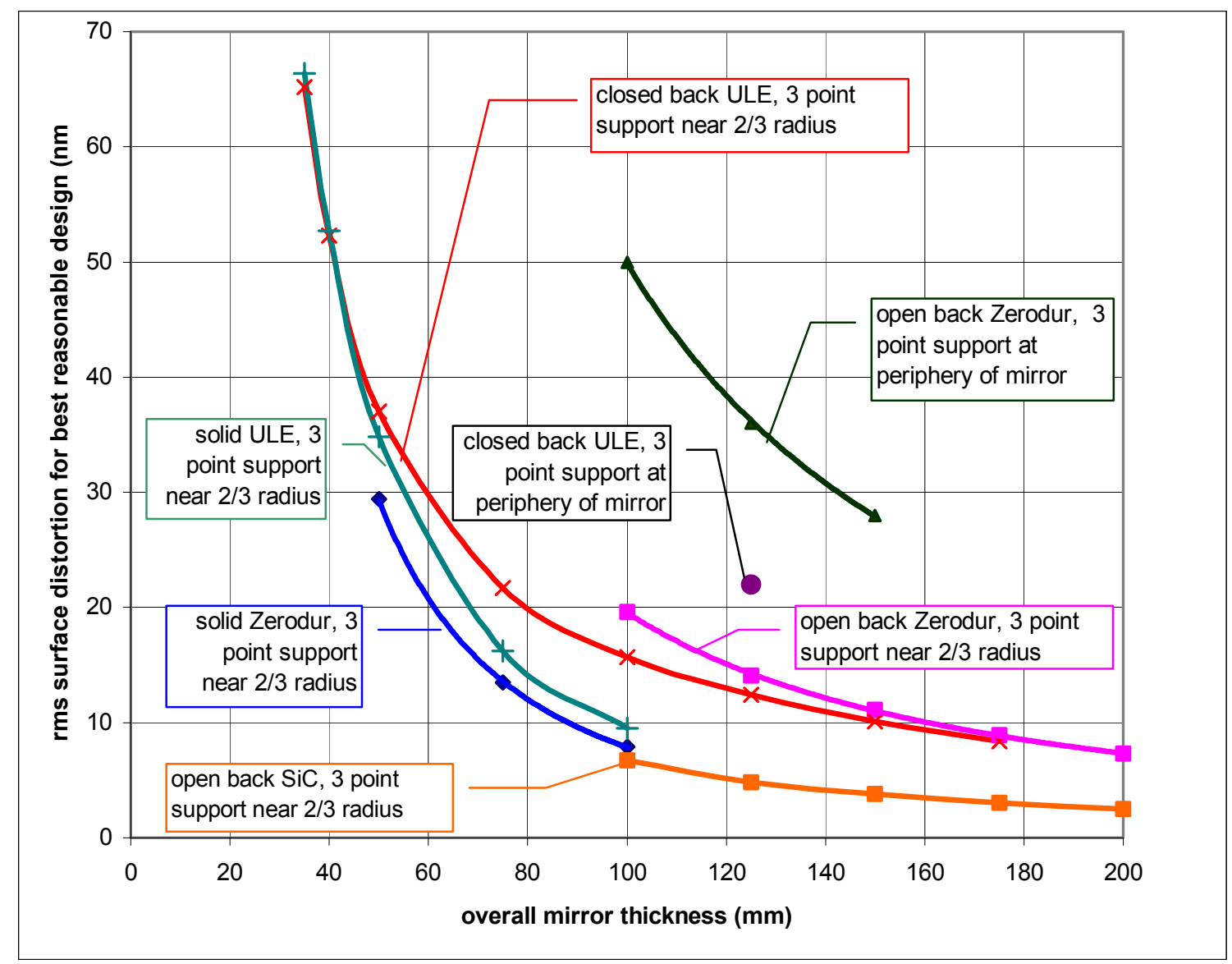

Figure 7: Examples of candidate secondary mirror FEM distortions in 1-G supported for vertical beam testing by three support bipods (from Besuner et al, in preparation).

In Figure 7 we show examples of RMS deformations of the secondary mirror for a variety of mirror designs, both solid and lightweighted. All examples have a common diameter of $451 \mathrm{~mm}$. The open-back Zerodur examples have $126 \mathrm{~mm}$ triangular cells, $6 \mathrm{~mm}$ thick faces and ribs. The closed back ULE examples have $6 \mathrm{~mm}$ thick faces and various rib thicknesses. One other example is a silicon carbide mirror, 
geometrically identical to the Zerodur cases but corrected for density and modulus, shown for comparison. The deformations can be small enough that they can be budgeted within the system acceptance tests without needing gravity unloading mechanisms. A comprehensive discussion is being prepared by Besuner et al (in preparation).

One built-in test feature we are exploring is the inclusion of a group of sub-aperture mirrors facing the primary mirror, located within the forward door or attached to the secondary support legs. To use these we would include a number of optical fiber point-source illuminators coplanar with the image sensors on the focal plane. The fine-guider CCD sensors in the focal plane run at a high frame rate and are operable at room temperature without cooling. As long as the air currents within the outer baffle are sufficiently calm, this built-in two-pass optical test setup should yield reproducible estimates of the wavefront tilt at a few pupil zones. This arrangement would allow us to perform a functional optical test at a number of occasions in the integration flow process.

\section{CONCLUSIONS}

We have presented an overview of the requirements and status of a telescope design for the planned SNAP mission. The optical, mechanical, and thermal studies and analyses conducted to date indicate that this telescope is manufacturable and testable using proven techniques. Upcoming work during the SNAP research and development phase will further refine these concepts.

\section{ACKNOWLEDGMENTS}

This work was supported by the Director, Office of Science, of the U.S. Department of Energy under Contract No. DE-AC03-76SF00098.

\section{REFERENCES}

1. S. Perlmutter et al. Astrophys. J., 517, p.565, 1999.

2. A.G. Riess et al, Astron. J., 116, p.1009. 1998.

3. G. Aldering et al, Proc. SPIE $4835 \# 21,2002$.

4. A. Kim et al, Proc. SPIE 4836 \#10, 2002.

5. Various authors, Session 97 "Probing Dark Energy with SNAP" and Session 126 "SNAP", 201st Meeting of the AAS, Seattle, Jan 2003.

6. M. Lampton et al, Proc. SPIE 4849 pp.215-226, 2002.

7. M.Lampton et al, Proc. SPIE 4854, 2002.

8. C.Bebek et al, Proc. SPIE $5164 \# 10,2003$.

9. A. Ealet et al, Proc. SPIE 4850 \#165, 2002.

10. D. Korsch, Appl.Optics 11, p.2986, 1972; 16, p.2074, 1977; 19, p.3640, 1980.

11. G. Bernstein, PASP 114 p.98, 2001.

Contact: mlampton@SSL.berkeley.edu; tel (USA) 510-642-3576 or 510-486-5047; Space Sciences Lab, University of California, Berkeley CA 94720 USA. 